\title{
Polyether Synthesis by Bulk Self-Condensation of Diols Catalyzed by Non-eutectic Acid Base Organocatalysts
}

\author{
Andere Basterretxea ${ }^{1}$, Elena Gabirondo ${ }^{1}$, Coralie Jehanno ${ }^{1}$, Haijin Zhu ${ }^{3}$, Irma Flores ${ }^{1}$, Alejandro J. \\ Müller $^{1,2}$, Agustin Etxeberria ${ }^{1}$, David Mecerreyes ${ }^{1,2}$, Olivier Coulembier ${ }^{4}$, Haritz Sardon ${ }^{1 *}$ \\ ${ }^{1}$ POLYMAT and Polymer Science and Technology Department, Faculty of Chemistry, University of the Basque Country \\ UPV/EHU, Paseo Manuel de Lardizabal, 3, 20018 Donostia-San Sebastián, Spain \\ ${ }^{2}$ Ikerbasque, Basque Foundation for Science, E-48011 Bilbao, Spain \\ ${ }^{3}$ Deakin University Burwood Campus, Institute for Frontier Materials, 221 Burwood Highway, VIC 3125, Australia \\ ${ }^{4}$ Center of Innovation and Research in Materials and Polymers (CIRMAP), Laboratory of Polymeric and Composite Materials, \\ University of Mons, Place du Parc 23, 7000 Mons, Belgium
}

KEYWORDS Aliphatic polyether, organocatalyst, polycondensation, non-eutectic acid-base organocatalysts, copolyether.

\begin{abstract}
Polyethers constitute a well-established class of polymers covering a wide range of applications from industrial manufacturing to nanomedicine. Nevertheless, their industrial implementation is limited to short chain aliphatic polyethers such as polyethyeleglycol (PEO or PEG), polypropyleneglycol (PPG) or polytetramethylenglycol (PTMG) produced by the ring-opening polymerization of the corresponding cyclic ethers. Herein we report a sustainable and scalable approach for the preparation of medium and long chain aliphatic polyethers by the melt self-polycondensation of aliphatic diols in the presence of non-eutectic acid base mixtures as organocatalyst. These organocatalysts were prepared by forming stoichiometric and non-stoichiometric complexes of methanesulfonic acid (MSA) and 1,5,7-triazabicyclo[4.4.0]dec-5-ene (TBD) as confirmed by NMR spectroscopy and DFT calculations. The non-stoichiometric 2:1 and 3:1 MSA:TBD molar complexes showed superior thermal stability. These non-eutectic acid base mixtures were tested in the bulk-self condensation of 1,6-hexanediol leading to telechelic $\alpha, \omega-$ hydroxy-poly(oxyhexane). The optimized polymerization conditions involved the use of MSA:TBD (3:1) catalyst in a three step polycondensation process at $130{ }^{\circ} \mathrm{C}$ $-180^{\circ} \mathrm{C}$ and $200{ }^{\circ} \mathrm{C}$ respectively. These conditions were applied to the synthesis of a wide range of aliphatic polyethers with a number of methylene units ranging from 6 to 12 units and molecular weights between 5,000 and 22,000 $\mathrm{g} \mathrm{mol}^{-1}$. The aliphatic polyethers were highly semicrystalline with melting temperatures ranging from 55 to $85{ }^{\circ} \mathrm{C}$. The synthesis approach was extended to the preparation of value-added copolymers from different lenght chain diols and different functionality, giving rise to different copolymer architectures from linear copolyethers to polyether thermosets. Altogether, this straightforward polymerization strategy enables the access to medium-long chain and cross-linked aliphatic polyethers using easily prepared and recyclable organocatalysts.
\end{abstract}

Polyethers are an important polymer family due to their versatile syntheses, thermal and chemical stability, and multiple applications. ${ }^{1,2}$ Polyethers such as as polyethyeleglycol (PEO or PEG), polypropyleneglycol (PPG) or polytetramethylenglycol (PTMG) are nowadays used in many different applications ranging from surfactants, automotive industry, batteries, food and cosmetic industry, nanomedicine or as soft segment in polyurethane chemistry. ${ }^{1,3-5}$ These polyethers are mainly produced by ring-opening polymerization of the corresponding cyclic ethers, such as oxiranes, oxetanes or tetrahydrofurane. ${ }^{6-8}$ However, using the aforementioned method, larger size polyethers (containing 6 or more methylene units) cannot be obtained because of the extreme stability of the cyclic ethers. In these cases, the production of longer methylene unit polyethers is achieved by polycondensation using the Williamson ether synthesis. ${ }^{9}$ In this case, the ether linkage is generated by a nucleophilic substitution of an alkoxide on an alkylating reagent (typically a halogenated alkane). Although this method is highly efficient, chloride is generated as side product during the polymerization which may generate hydrocholic acid and detracts from the sustainable production of these polymers. Very recently, Meier et al. prepared polyethers by the reduction of polyesters in the presence of tetramethyldisiloxane and $\mathrm{GaBr}_{3}$ catalyst in a more sustainable process, but this process requires the previous preparation of the analogous polyester. ${ }^{10} \mathrm{An}$ old but not as explored route to produce polyethers is the acid catalyzed self-condensation of alcohols resulting in an ether bond. In the 1950s, Rhoad and Flory pioneered the self condensation of 1,10-decanediol in the presence of sulfamic or sulfuric acids at elevated temperature $\left(300{ }^{\circ} \mathrm{C}\right)$ in bulk polymerization conditions. ${ }^{11}$ Almost twenty years later, Kobayashi et al., used the same method to synthesize linear poly(oxyalkylenes) in the presence of $\mathrm{H}_{2} \mathrm{SO}_{4}$ and $\left(\mathrm{C}_{2} \mathrm{H}_{5}\right)_{2} \mathrm{O} \cdot \mathrm{BF}_{3} .{ }^{12}$ As this reaction is reversible, care has to be taken when ethers are used under strong acidic conditions. Albeit the self-condensation processes in bulk is a simple and environmental friendly polymerization method, it presents a 
setback; since some acids are highly volatile and they can be decomposed during the polymerization process due to the harsh polymerization conditions. In 2011, Fradet et al. minimized these drawbacks using Brønsted Acid Ionic Liquids as solvent and catalysts which allowed the reactions to be carried out at lower temperatures $\left(130{ }^{\circ} \mathrm{C}\right){ }^{13}$ Brønsted Acid Ionic Liquids combine the catalytic activity of Brønsted Acid with the high thermal stability and low vapor pressure of ionic liquids. However, the ones employed by Fradet have prohibitive price and the side reactions occurring during the polymerization limited their potential. A cost-effective alternative to Brønsted Acid Ionic liquids are Protic Ionic Liquids/Salts which can be prepared through the simple proton transfer from a Brønsted acid to a Brønsted base. These protic ionic compounds have been shown to be suitable alternatives to classic organometallic catalysts. They smartly combine the excellent catalytic ability of organocatalysts with the thermal stability of ionic liquids, resisting degradation up to $>400{ }^{\circ} \mathrm{C} .{ }^{14}$ Thus, Flores et al. demonstrated that the Protic Ionic Salt 1,8-diazabicyclo[5.4.0]undec7-ene: benzoic acid (DBU:BA) was a competitive catalyst for PET synthesis showing a good stability and catalytic activity even at elevated temperatures up to $>250{ }^{\circ} \mathrm{C} .{ }^{15}$ Similarly, equimolar mixtures of 1,5,7-triazabicyclo[4.4.0]dec-5-ene (TBD) and methanesulfonic acid (MSA) proved to be an efficient and extremely thermally stable catalyst in the depolymerization of PET being even able to be recycled several times. ${ }^{14}$ One of the key features of protic ionic compounds is their ability to form stable complexes even using non-equimolar mixtures by H-bonding interaction between the acid and base preparing the non-eutectic acid base mixtures. Taking advantage of this unique characteristic, Del monte et al. prepared different non-equimolar mixtures of methanesulfonic acid (MSA) and the 1,5,7-triazabicyclo[4.4.0]dec-5-ene (TBD) for the ring opening polymerization (ROP) of $\varepsilon$-caprolactone. ${ }^{16}$

In this work, we benefited from the versatility and easy synthesis of acid base mixtures for investigating the melt polymerization of polyethers by self-condensation of diols. First, different equimolar and non-equimolar mixtures of MSA and TBD were prepared leading to protic ionic salt (1:1 mixture) and different non-eutectic acid base mixtuters which were characterized extensively. This catalyst system was investigated as it has shown some potential to work at elevated temperatures without suffering any degradation. The protic ionic salt and the-non-eutectic acid base organocatalysts were used in a second step for investigating the polyetherification of 1,6-hexanediol. We found that some non-eutectic acid base organocatalysts were highly efficient for the polymerization process and also thermally stable, which allowed the recovery and the subsequent recycling of the catalyst. This synthetic strategy was extended to seven other long chain diols which yielded semicrystalline aliphatic polyethers whose crystalline structure was also determined. To finish, we expanded the frontiers of this work by copolymerizing different long chain diols and functionality resulting in both linear and crosslinked copolyethers.

\section{Experimental Section}

\section{Materials}

1,12-dodecanediol (99\%, Sigma-Aldrich), 1,11-undecanediol (98\%, ABCR), 1,10-decanediol (98\%, Sigma Aldrich), 1,9nonanediol (98\%, Sigma-Aldrich), 1,8-octanediol (98\%,
Sigma-Aldrich), 1,7-heptanediol (95\%, Sigma-Aldrich), 1,6hexanediol (99\% Sigma-Aldrich), 1,5-pentanediol (96\% Sigma-Aldrich) and 1,4-butanediol (99\% Sigma-Aldrich) were used as received after being dried in Toluene. Methanesulfonic acid (MSA, 99\%) and 1,5,7-Triazabicyclo[4.4.0]dec-5-ene (TBD, 98\%), glycerol (99\%) phenyl isocyanate (98\%), chloroform $\left(\mathrm{CDCl}_{3}\right)$, methanol $\left(\mathrm{CH}_{3} \mathrm{OH}\right)$ and the rest of the solvents used on this work were supplied by Sigma-Aldrich and used as received.

\section{Preparation of the catalyst mixtures}

Different dual catalysts were prepared by mixing Methanesulfonic acid (MSA) and 1,5,7-Triazabicyclo[4.4.0]dec-5-ene (TBD) at different molar ratios; $3: 1\left(0.1 \mathrm{~g}, 1.0410^{-3} \mathrm{~mol}\right.$ of MSA and $0.048 \mathrm{~g}, 3.4710^{-4} \mathrm{~mol}$ of TBD), 2:1 (0.09 g, $9.3610^{-}$ ${ }^{4} \mathrm{~mol}$ of MSA and $0.065 \mathrm{~g}, 4.6810^{-4} \mathrm{~mol}$ of TBD), $1: 1(0.07 \mathrm{~g}$, $7.2810^{-4} \mathrm{~mol}$ of MSA and $0.101 \mathrm{~g}, 7.2810^{-4} \mathrm{~mol}$ of TBD), $1: 2$ $\left(0.04 \mathrm{~g}, 4.1610^{-4} \mathrm{~mol}\right.$ of MSA and $0.116 \mathrm{~g}, 8.3210^{-4} \mathrm{~mol}$ of TBD), 1:3 (0.03 g, $3.1210^{-4} \mathrm{~mol}$ of MSA and $0.130 \mathrm{~g}, 9.3610^{-}$ ${ }^{4} \mathrm{~mol}$ of TBD). Afterwards, the mixtures were thermally treated at $90^{\circ} \mathrm{C}$ over 30 minutes under stirring until complete formation of homogeneous and transparent liquid solution or salt.

\section{Characterization of the catalysts \\ ${ }^{1} \mathrm{H}$ and ${ }^{13} \mathrm{C}$ Nuclear Magnetic Resonance (NMR) spectros- copy}

${ }^{1} \mathrm{H}$ and ${ }^{13} \mathrm{C}$ Nuclear Magnetic Resonance (NMR) spectroscopy were recorded in a Bruker Avance DPX 300 at $300.16 \mathrm{MHz}$ and at $75.5 \mathrm{MHz}$ of resonance frequency respectively, using deuterated chloroform $\left(\mathrm{CDCl}_{3}\right)$ as solvent at room temperature. Experimental conditions were as follows: a) for ${ }^{1} \mathrm{H}$ NMR spectroscopy: $10 \mathrm{mg}$ of sample; $3 \mathrm{~s}$ acquisition time; $1 \mathrm{~s}$ delay time; 8.5 $\mu$ s pulse; spectral width $5000 \mathrm{~Hz}$ and 32 scans; b) for ${ }^{13} \mathrm{C}$ NMR spectroscopy: $40 \mathrm{mg} ; 3 \mathrm{~s}$ acquisition time; 4 s delay time; 5.5 $\mu$ s pulse; spectral width $18800 \mathrm{~Hz}$ and more than 10000 scans.

\section{Thermogravimetric analyses (TGA)}

Thermogravimetric analyses (TGA) were carried out using a Q500 Thermogravimetric Analyzer from TA Instruments. Samples were heated from room temperature to $600{ }^{\circ} \mathrm{C}$ at a rate of $10{ }^{\circ} \mathrm{C} /$ min under a constant $\mathrm{N} 2$ flow.

\section{Density functional theory (DFT)}

The electronic structure calculations were carried out using the Gaussian 09 suite of programs ${ }^{17}$. Geometry optimization were performed using $\omega B$ 97XD functional with the $6-31+G(d, p)$ basis set. Vibrational frequencies were calculated at the same level of theory to ensure that the optimized structures were minima in the potential energy surface (no imaginery frequencies) and to determine the zero-point vibrational energy (ZPVE) and the thermal vibrational corrections at $\mathrm{T}=298 \mathrm{~K}$. Single-point energy calculations were performed at the $\omega$ B97XD/6-311++ $\mathrm{G}(2 \mathrm{df}, 2 \mathrm{p})$ level of theory in order to refine the electronic energy.

\section{Pulsed field gradient NMR (PFG-NMR)}


Pulsed field gradient NMR (PFG-NMR)measurements were performed on a $500 \mathrm{MHz}$ standard-bore Bruker Avance III solution-sate NMR spectrometer. The sample was packed in a 5 $\mathrm{mm}$ Schott E NMR tube to a height of $5 \mathrm{~cm}$. Pulse gradient stimulated echo (PGSTE) was used for diffusion measurements. The gradient pulse duration was $10 \mathrm{~ms}$, diffusion time was 50 $\mathrm{ms}$, and gradient strength was varied between $0.5 \sim 100$ Gaussian/m in a $\log$ scale to have the full NMR signal attenuation. Recycle delay was $2 \mathrm{~s}$.

\section{Bulk Self-condensation of 1,6-hexanediol using the catalyst mixtures}

The different MSA:TBD complexes were tested in the self-condensation of 1,6-hexanediol. For that 0.05 equiv. ( $5 \mathrm{~mol} \%, 7.45$ $10^{-4} \mathrm{~mol}$ ) of previously prepared protic ionic salt and non-eutectic acid base organocatalysts were mixed with $1.76 \mathrm{~g}(0.015$ mol) of 1,6-hexanediol in a $25 \mathrm{ml}$ Schlenk tube with a magnetic stirred. The sealed reaction vessel was submerged into a preheated oil bath at $180{ }^{\circ} \mathrm{C}(72 \mathrm{~h})$ under vacuum. The reaction was stopped by rapid cooling in liquid nitrogen. For the purification, the samples were dissolved in chloroform and precipitate in cold methanol. The resulted polyethers were filtrated and dried under vacuum at RT for $24 \mathrm{~h}$ before their characterization.

The synthesis of the $\approx 200 \mathrm{~g}$ of polyether was carried out in a $500 \mathrm{ml}$ reactor using an electrical stirred. For that $200 \mathrm{~g}$ of 1,6hexanediol ( $1.692 \mathrm{~mol}$ ) was mixed with a 0.05 equiv. ( $5 \mathrm{~mol} \%)$ of previously prepared non-eutectic acid base organocatalyst 3:1 (0.084 mol, $36.179 \mathrm{~g})$. The reactor was submerged into a pre-heated oil bath at $130{ }^{\circ} \mathrm{C}(24 \mathrm{~h}), 180{ }^{\circ} \mathrm{C}(24 \mathrm{~h})$ and $200{ }^{\circ} \mathrm{C}$ (24 h) under vacuum. The reaction was stopped by rapid cooling in liquid nitrogen.

\section{Catalyst recycling}

The reaction was carried out mixing 0.05 equiv. ( $5 \mathrm{~mol} \%, 7.45$ $\left.10^{-4} \mathrm{~mol}, 0.318 \mathrm{~g}\right)$ of the non-eutectic MSA:TBD 3:1 with 1.760 $\mathrm{g}(0.015 \mathrm{~mol})$ of 1,6-hexanediol in bulk. After performing the polymerization the polymer-catalyst mixture was dissolved in chloroform and precipitated in cold methanol where all the polymer was precipitated. The catalyst containing filtrate was concentrated and recrystrallized from cold hexane to afford the initial catalyst (which was verified by ${ }^{1} \mathrm{H}$ NMR) (93\% yield). The catalyst was reused again to confirm its potential to be recycled.

\section{Bulk Self-condensation of different aliphatic diols}

The self-condensation of the diols were accomplished using as catalyst the non-eutectic acid base mixtures formed by the molar mixture of MSA:TBD 3:1. For that, 0.05 equiv. (5 mol \%) of MSA:TBD 3:1(7.45 $\left.10^{-4} \mathrm{~mol}, 0.318 \mathrm{~g}\right)$ was mixed with 0.015 mol of the corresponding diol (3.013 $\mathrm{g}$ of 1,12-dodecanediol, $2.805 \mathrm{~g}$ of 1,11-undecanediol, $2.596 \mathrm{~g}$ of 1,10-decanediol, $2.387 \mathrm{~g}$ of 1,9-nonanediol, $2.178 \mathrm{~g}$ of 1,8-octanediol, $1.969 \mathrm{~g}$ of 1,7-heptanediol and $1.760 \mathrm{~g}$ of 1,6-hexanediol) in a the Schlenk flask with a magnetic stirrer. The sealed reaction vessel was submerged into a pre-heated oil bath at $130{ }^{\circ} \mathrm{C}(24 \mathrm{~h}), 180{ }^{\circ} \mathrm{C}$ $(24 \mathrm{~h})$ and $200{ }^{\circ} \mathrm{C}(24 \mathrm{~h})$ under vacuum. The reaction was stopped by rapid cooling in liquid nitrogen.

For the purification, the samples were dissolved in chloroform and precipitated in cold methanol. The resulted polyethers were filtrated and dried under vacuum at RT for $24 \mathrm{~h}$ before their characterization.

Bulk Self-condensation copolymerization of 1,6-hexanediol/1-12-dodecanediol and 1,6-hexanediol/glycerol

Copolymers were synthesized by self-condensation of the respective monomers; 1,6-hexanediol, 1,12-dodecanediol and glycerol. For that, 0.05 equiv. $(5 \mathrm{~mol} \%)$ of the non-eutectic acid base mixture MSA:TBD 3:1 was prepared as it was previously described. After that, a mixture of monomers containing 1,6hexanediol/1,12-dodecanediol 50/50 (1.48 g, $0.012 \mathrm{~mol} / 2.534$ $\mathrm{g}, 0.012 \mathrm{~mol})$ and 1,6-hexanediol/glycerol 50/50 (1.48 g, 0.012 $\mathrm{mol} / 1.153 \mathrm{~g}, 0.012 \mathrm{~mol}$ )was added respectively to the reaction vessel. Then, the sealed reaction vessel was submerged into a pre-heated oil bath at $130{ }^{\circ} \mathrm{C}(24 \mathrm{~h}), 180{ }^{\circ} \mathrm{C}(24 \mathrm{~h})$ and $200{ }^{\circ} \mathrm{C}$ $(24 \mathrm{~h})$ under vacuum. The reaction was stopped by cooling up at room temperature. For the purification, the copolyether 1,6hexanediol-co-1,12-dodecanediol was dissolved in chloroform and precipitate in cold methanol. Then the sample was filtrated and dried under vacuum at RT for $24 \mathrm{~h}$ before their characterization. The copolyether 1,6-hexanediol-co-glycerol was washed with chloroform.

\section{Result and Discussion}

\section{Characterization of the acid base organocatalysts based on MSA and TBD}

First, MSA and TBD based organocatalysts were prepared in different molar ratios such as $3: 1,2: 1$ and $1: 1$ by simple mixing at $90{ }^{\circ} \mathrm{C}$ for 30 minutes until obtaining a transparent and homogeneous organocatalyst. In order to confirm the formation of the acid base organocatalysts the resulting mixtures were characterized by ${ }^{1} \mathrm{H}$ NMR spectroscopy in DMSO, while their thermal degradation was investigated with TGA. ${ }^{14,16}$ 
(a)

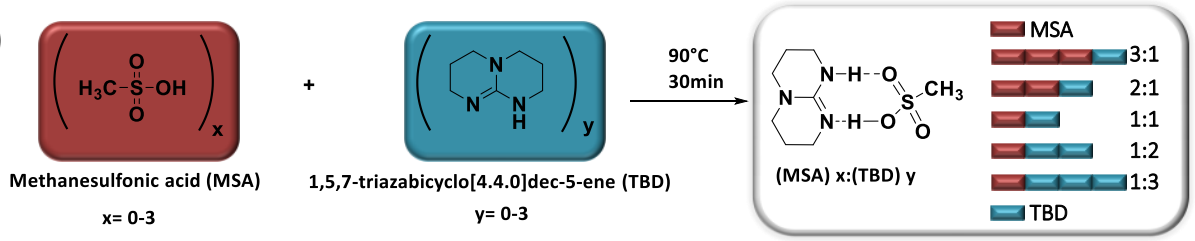

(b)

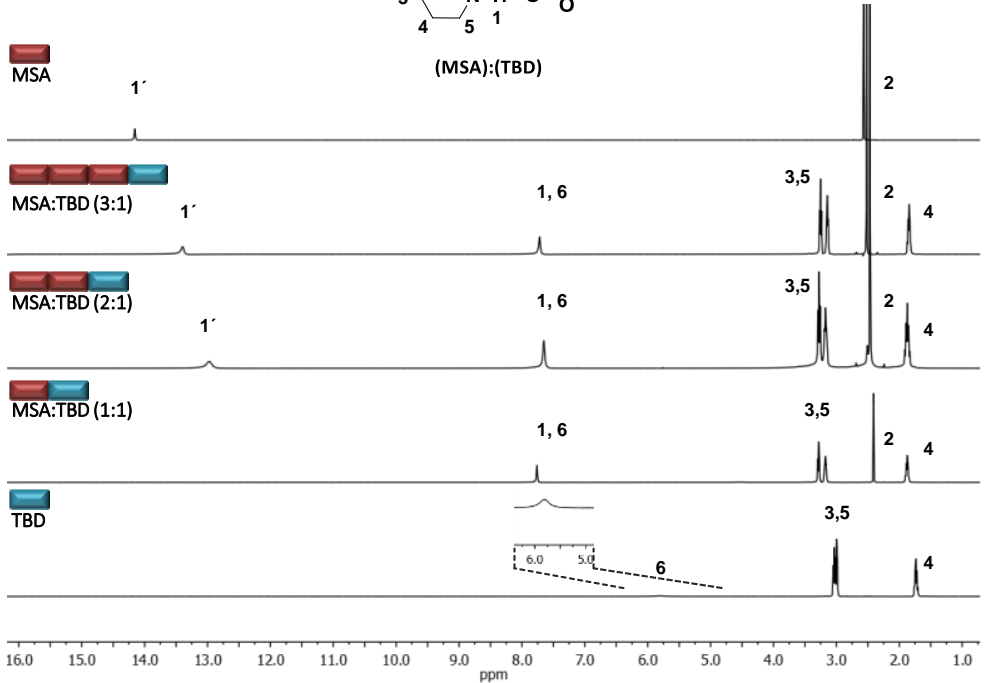

(c)

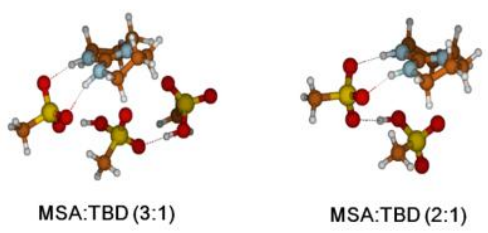

(d)

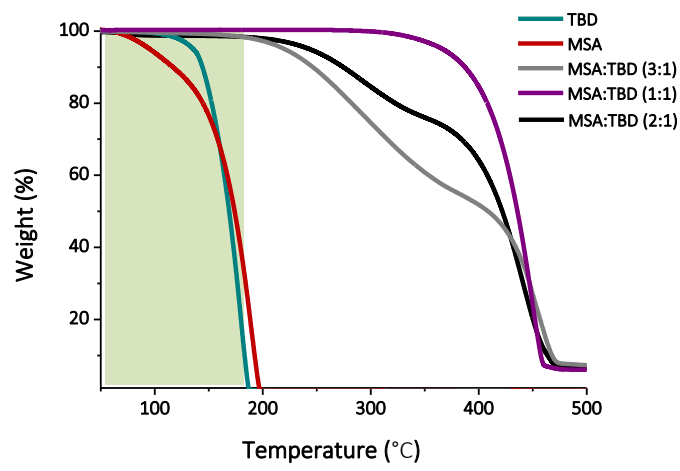

Figure 1. a) Scheme of the synthesis of the protic ionic compound (1:1) and the non-eutectic acid base organocatalysts (3:1 \& 2:1), b) ${ }^{1} \mathrm{H}$ NMR spectroscopy in DMSO of the catalyst used for the polyetherification, c) calculated structures of MSA:TBD (2:1) and (3:1) and d) Thermogravimetric analysis of methanesulfonic acid (MSA), 1,5,7-triazabicyclo[4.4.0]dec-5-ene (TBD) and the acid base mixtures $(3: 1,2: 1$ and $1: 1)$.

The recorded spectra for pure TBD, MSA shows the characteristic N-H proton signal of TBD at $\delta=5.81 \mathrm{ppm}$ and signal of MSA acid at $\delta=14.16 \mathrm{ppm}$. In contrast, for the (1:1) mixture, these two signals disappear and a new one integrating for 2 protons appears at $\delta=7.71 \mathrm{ppm}$, which demonstrates the formation of the non-eutectic acid base mixtures by a proton transfer from MSA to TBD (Figure 1b). In the case of non-equimolar mixtures together with this band at $7.71 \mathrm{ppm}$ we found the peak of the acid proton which was shifted to lower values $(13.0 \mathrm{ppm}$ and $13.5 \mathrm{ppm}$ for the 2:1 and 3:1 mixtures respectively). The appearance of this chemical shift in the ${ }^{1} \mathrm{H}$ NMR spectra of the mixtures as compared to those of the pure components confirms also the presence of $\mathrm{H}$-bond complexes between non-equimolar mixtures of MSA and TBD (Figure 1b). To further understand the complex structures of the non-equimolar mixtures, the variable temperature ${ }^{1} \mathrm{H}$ NMR spectra of the 3:1 mixture were measured in its dry state (without DMSO solvent), and results are shown in SI section. At $-10{ }^{\circ} \mathrm{C}$, two peaks which were attributed to the acid proton of MSA (left side) and the N-H of the TBD (right side) can be clearly distinguished. The integrations of both peaks are roughly identical, suggesting that in this $3: 1$ mixture, one third of the MSA acid protons transferred to the $\mathrm{TBD}$, forming a $[\mathrm{TBD}]^{+}[\mathrm{MSA}]^{-}$salt. Whereas the remaining two thirds of MSA acid protons remain with the MSA molecules in the mixture. More interestingly, the broad feature of both proton lines suggests fast proton exchange between both proton species, at the timescale of tens to hundreds of milliseconds. More specifically, a dynamic equilibrium between the MSA acid and the $[\mathrm{TBD}]^{+}[\mathrm{MSA}]^{-}$salt exists; where the dwelling time of each proton state is in the order of hundreds of milliseconds at $-10^{\circ} \mathrm{C}$, and is highly dependent on the temperature.

As shown in Figure S2, an increase of temperature from -10 to $100{ }^{\circ} \mathrm{C}$ leads to a significant line broadening at first, then a gradual merging of both lines into a single broad line, and finally a narrowing of the single line. These variation of the proton line widths and positions with temperature is a typical feature and consequence of chemical exchange process between two proton spieces, and has been observed in many ionic liquids and other systems. ${ }^{18,19}$ It is worth mentioning that, at $40{ }^{\circ} \mathrm{C}$ and above, the two proton peaks merged into a single peak, indicating that at the NMR observation time scale of tens of milliseconds, the proton exchange is fast enough to average out the environmental differences and the acid so that the $\mathrm{N}-\mathrm{H}$ protons are equivalent. In order to get better insights into the molecular structures of MSA:TBD (2:1) and (3:1), both complexes have been investigated by means of quantum chemical calculations using $\omega \mathrm{B} 97 \mathrm{XD} / 6-311++\mathrm{G}(2 \mathrm{df}, 2 \mathrm{p})$ method and the resulting stable complexes are presented in Figure 1c. Through these calculations, we found that in the case of 2:1 complex, not only does one of the acidic proton of MSA 1 completely transfer to the basic nitrogen of TBD, thereby forming a hydrogen bond between the N-H moiety of TBD and one of the oxygens of the sulfonyl group of $\mathrm{MSA}_{1}$, but also that the acidic proton of $\mathrm{MSA}_{2}$ 
forms a hydrogen bond with the sulfonyl group of MSA1. These observations support the chemical shift of 1' (corresponding to the acidic proton of MSA) in the ${ }^{1} \mathrm{H}$ NMR spectra and occurs thanks to the dual character (nucleophilic \& electrophilic) of organic acids. $^{20}$

Similar results were obtained for the 3:1 non-eutectic acid base complex, as $\mathrm{MSA}_{3}$ is also linked to the complex through the transfer of its acidic proton to MSA2. To further confirm the complex formation, the thermal-degradation profiles were studied by TGA (Figure 1d). While TBD and MSA present a relatively low degradation temperature, with $50 \%$ of the mass lost before $180{ }^{\circ} \mathrm{C}$ for both molecules $\left(\mathrm{T}_{50 \%}=170{ }^{\circ} \mathrm{C}\right.$ for TBD and $\mathrm{T}_{50 \%}=174{ }^{\circ} \mathrm{C}$ for MSA), samples $(2: 1)$ and (3:1) show a two step degradation profile at higher temperatures. A first deterioration of the catalysts occurs after $200{ }^{\circ} \mathrm{C}$, loosing $25 \%$ of weight for (2:1) and $45 \%$ for (3:1). Those losses could correspond to the releasing of one and two molecules of MSA, respectively for (2:1) and (3:1). Therefore, the second degradation event corresponds to the already reported decomposition of MSA:TBD $(1: 1)$ with $\mathrm{T}_{50 \%}=438^{\circ} \mathrm{C}$ (Figure S23-S25). ${ }^{14}$ These results revealed that the mixtures $(3: 1$ and $2: 1)$ were better resisting to temperature than MSA or TBD as individual components, which is in good agreement with the high dissociation energies encountered using DFT calculations of the non-eutectic acid base mixtures.

\section{Catalytic activity testing and optimization of the self-con- densation temperature using 1,6-hexanediol}

The different MSA:TBD organocatalysts were tested in the polyetherification of 1,6-hexanediol using 0.05 equiv. of organocatalyst at $180{ }^{\circ} \mathrm{C}$ under solvent free conditions (Figure 2a, Table 1). The polymerizations were monitored using ${ }^{1} \mathrm{H}$ NMR, by the diagnostic disappearance of 1,6-hexanediol hydroxyl methylene protons (red signal at $\delta 3.65 \mathrm{ppm}$, adjacent to the alcohol) and their subsequent reappearance at $\delta 3.33 \mathrm{ppm}$ due to ether formation (grey signal) (Figure 2c).

As the molecular weights were relatively low, they were determined by NMR in order to compare all the samples as some of the samples were out of the detection limit of the SEC equipment. To do so, the polyether was end-capped with phenyl isocyanate not only to identify better the end-groups and to determine the molecular weight by NMR but also to confirm the presence of linear structures. As expected the proton adjacent to the capped alcohol shifted to higher ppm ensuring the presence of alcohol end groups and ensuring not any overlapping with internal ether groups which facilitates the molecular weight measurements (SI section). The reaction was found to be dramatically catalyst dependent (Figure 2d). As expected, when pristine TBD alone was used no monomer conversion was observed (entry 1). Meanwhile, when using pure MSA as catalyst the monomer conversion was $46 \mathrm{~mol}$. \% after $72 \mathrm{~h}$ (entry 2). This result confirms the catalytic activity of organic acids to mediate the self-condensation of alcohols. Nevertheless, in the presence of MSA the polymerization turned brownish color. We believe that the harsh conditions required for the polymerization reaction leads to degradation of the MSA catalyst thereby preventing its catalytic action as was demonstrated by Jehanno et al. in a similar study. ${ }^{14}$ Conversely, non-eutectic acid base mixtures containing excess MSA (2:1 and 3:1) showed very efficient catalytic activity and monomer conversions above $95 \%$ were obtained resulting in polyether of 5,200 $\mathrm{g} \mathrm{mol}^{-1}$ and 4,500 $\mathrm{g} \mathrm{mol}^{-1}$ respectively (entry 3,4 ).

Surprisingly, the obtained polymers were completely white suggesting that these two catalysts were not degrading the polymerization process. In order to confirm the importance of acid excess in the polymerization two control experiments were run with excess of TBD (MSA:TBD ration 1:2 \& 1:3 respectively) (entry 6 and 7). We observed much lower monomer conversion (less than 20\%) as this reaction is known to be acid catalyzed.

In order to achieve high molecular weight the influence of the temperature on the polymerization reaction was investigated. After screening different polymerization temperatures (entry 3, 8 and 9), we found that the highest molecular weights were obtained via polymerizing the 1,6 -hexanediol at $180{ }^{\circ} \mathrm{C}(\mathrm{Mn}=$ $5200 \mathrm{~g} \mathrm{~mol}^{-1}$ ). While using lower temperatures. i.e. $150{ }^{\circ} \mathrm{C}$ and $130^{\circ} \mathrm{C}$, the attained molecular weights were substantially lower $\left(2,400\right.$ and $1,300 \mathrm{~g} \mathrm{~mol}^{-1}$ respectively). Nevertheless, we found that when running the polymerization at $180{ }^{\circ} \mathrm{C}$ the associated yield was lower in comparison to $150{ }^{\circ} \mathrm{C}$ and $130{ }^{\circ} \mathrm{C}$ due approaching the boiling point of the monomer at the early hours of the polymerization process. In order to avoid monomer evaporation while polymerization was occurring, the polymerization was undertaken in various steps, mimicking the strategy used in other polycondensation processes such as PET or polycarbonates. ${ }^{3}$ Thus the same reaction was carried out first at $130{ }^{\circ} \mathrm{C}$ for $24 \mathrm{~h}$, after which the temperature was raised to $180{ }^{\circ} \mathrm{C}$ for $48 \mathrm{~h}$. In this particular case, besides not observing any monomer evaporation during polymerization a higher molecular weight polyether $\left(7,000 \mathrm{~g} \mathrm{~mol}^{-1}\right)$ was obtained (entry 10). 
(a)

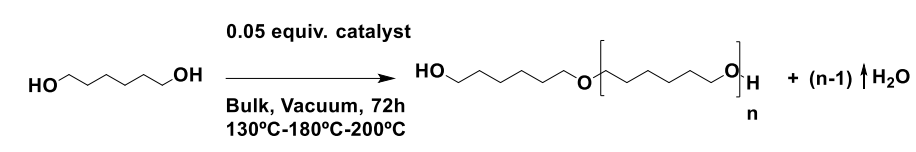

(b)

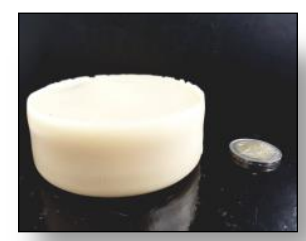

(e) (c)

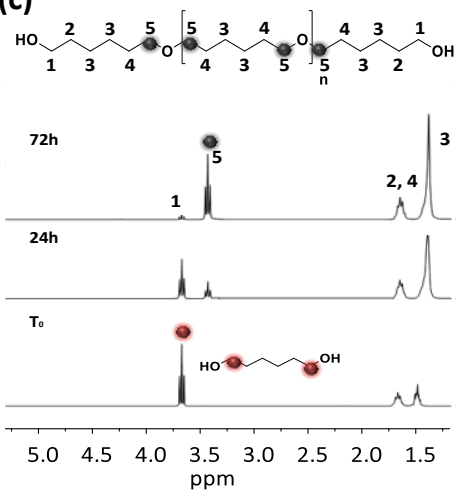

(d)

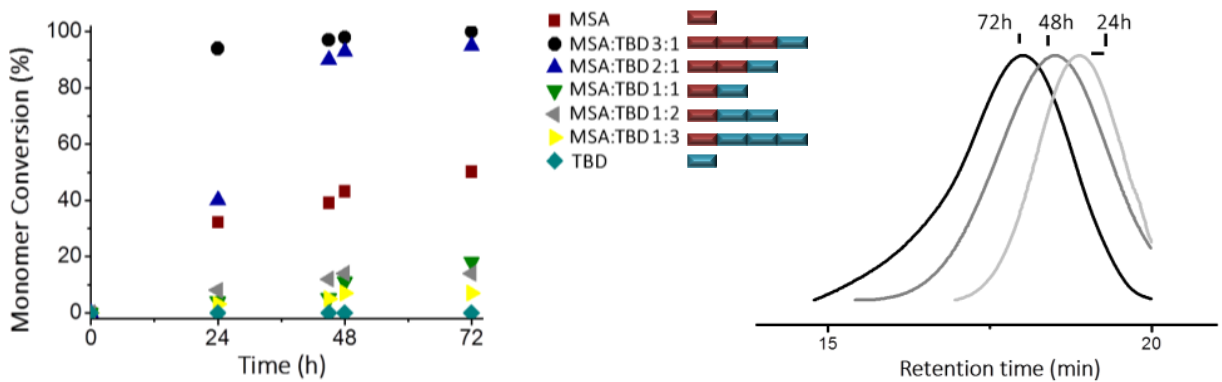

Figure 2. a) Scheme of the self-condensation reaction of 1,6-hexanediol catalyzed using different catalysts, b) ${ }^{1} \mathrm{H}$ NMR spectra of the reaction media during the course of the reaction, c)monomer conversion values (\%) at 24, 48 and $72 \mathrm{~h}$ reaction using different ratios of MSA:TBD respectively as catalysts and d)SEC chromatograms at different reaction times.

Table 1. Screened data of the optimization of the polyetherification of 1,6-hexanediol using different MSA:TBD at different molar ratios as catalyst and different temperatures.

\begin{tabular}{|c|c|c|c|c|c|c|c|}
\hline Entry & $\begin{array}{l}\text { MSA } \\
\text { (equiv.) }\end{array}$ & $\begin{array}{l}\text { TBD } \\
\text { (equiv.) }\end{array}$ & Monomer/catalyst & Temperature/ ${ }^{\circ} \mathrm{C}$ & Mon. Conv (\%)72h ${ }^{a}$ & $\operatorname{Mn}\left(\mathrm{g} \mathrm{mol}^{-1}\right)^{a}$ & Yield. $(\%)^{b}$ \\
\hline 1 & - & 1 & $1 / 0.05$ & 180 & 0 & - & - \\
\hline 2 & 1 & - & $1 / 0.05$ & 180 & 50 & 900 & 29 \\
\hline 3 & 3 & 1 & $1 / 0.05$ & 180 & 97 & 5200 & 43 \\
\hline 4 & 2 & 1 & $1 / 0.05$ & 180 & 95 & 4500 & 39 \\
\hline 5 & 1 & 1 & $1 / 0.05$ & 180 & 18 & -- & -- \\
\hline 6 & 1 & 2 & $1 / 0.05$ & 180 & 14 & -- & -- \\
\hline 7 & 1 & 3 & $1 / 0.05$ & 180 & 7 & -- & -- \\
\hline 8 & 3 & 1 & $1 / 0.05$ & 130 & 80 & 1300 & 58 \\
\hline 9 & 3 & 1 & $1 / 0.05$ & 150 & 86 & 2400 & 61 \\
\hline 10 & 3 & 1 & $1 / 0.05$ & *130-180 & 98 & 7000 & 73 \\
\hline 11 & 3 & 1 & $1 / 0.05$ & ${ }^{* *} 130-180-200$ & 98 & 11300 & 84 \\
\hline 12 & 3 & 1 & 1/0.05(recycled) & $* * 130-180-200$ & 97 & 10200 & 76 \\
\hline
\end{tabular}

${ }^{a}$ Determined by ${ }^{1} \mathrm{H}$ NMR spectroscopy. ${ }^{b}$ Isolated yield. ${ }^{*} 130{ }^{\circ} \mathrm{C}(24 \mathrm{~h})$ and $180^{\circ} \mathrm{C}(48 \mathrm{~h}){ }^{* *} 130{ }^{\mathrm{a}} \mathrm{C}(24 \mathrm{~h}), 180^{\circ} \mathrm{C}(24 \mathrm{~h})$ and $200{ }^{\circ} \mathrm{C}(24 \mathrm{~h})$.

A further polymerization step at $200{ }^{\circ} \mathrm{C}$ for $24 \mathrm{~h}$ (entry 11) revealed an increase of the molar mass of the final polyether $\left(11,300 \mathrm{~g} \mathrm{~mol}^{-1}\right)$ while maintaining high polymerization yields. Herein, the gradual increase of the temperature from $130{ }^{\circ} \mathrm{C}$ to $200{ }^{\circ} \mathrm{C}$ enables us to avoid the losses of monomer achieving higher yields and molecular weights (Figure 2e). Further increase of temperature and time did not significantly alter the molecular weights or the polymerization yields.
After screening several polymerization conditions, it was found that the best results in terms of molecular weight and conversions were obtained via a multi-step polymerization process (the first one at $130{ }^{\circ} \mathrm{C}$ for 24 , a second one at $180{ }^{\circ} \mathrm{C}$ for $24 \mathrm{~h}$ and a thirt one at $200{ }^{\circ} \mathrm{C}$ for $24 \mathrm{~h}$ ) in the presence of $5 \mathrm{~mol} \%$ of MSA:TBD (3:1) catalyst. In order to make the process even more sustainable, we intended to recycle the organocatalyst from the polymer and re-used in another polymerization. The catalyst was recovered near quantitatively from the polymer mixture during the purification process $(90 \%)$. To recover the 
catalyst, the polymer-catalyst mixture was dissolved in chloroform and precipitated in cold methanol where all the polymer was precipitated. The catalyst containing filtrate was concentrated and recrystrallized from hexane to afford the initial catalyst (which was verified by ${ }^{1} \mathrm{H}$ NMR). The catalyst was reused again (entry 12) confirming its potential to be recovered as the obtained polymer has similar molecular weights than using freshly prepared catalysts (entry 11). Interestingly, the reaction could be performed in $200 \mathrm{~g}$ scale without suffering any color change and similar yield and molecular weights due to the high stability of the organocatalyst as shown in the picture of the polyether obtained in Figure 2b.

Synthesis of aliphatic polyethers with different number of methylene units

Scheme 1. Synthesis route of the aliphatic polyethers from different aliphatic diols

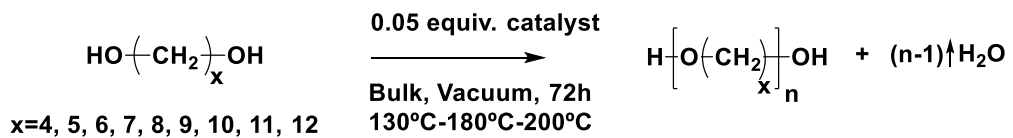

Table 2. Characterization data of the homopolymers synthesized from different aliphatic diols. in the presence of 0.05 equiv. of MSA:TBD (3:1) catalyst at $130-200^{\circ} \mathrm{C}$

\begin{tabular}{|c|c|c|c|c|c|c|c|}
\hline Monomer & $M_{n}\left(\mathrm{~g} \mathrm{~mol}^{-1}\right)^{a}$ & $\bigoplus^{a}$ & Yield $(\%)^{b}$ & $T_{m}\left({ }^{\circ} \mathrm{C}\right)^{c}$ & $\Delta H_{m}(\mathrm{~J} / \mathrm{g})^{c}$ & $2 \theta^{d}$ & $d(\mathrm{~nm})^{d}$ \\
\hline 1,6-hexanediol & 22000 & 1.9 & 70 & 54.9 & 83 & 16.04 & 0.369 \\
\hline 1,7-heptanediol & 8000 & 2.3 & 57 & 65.5 & 134 & 15.9 & 0.372 \\
\hline 1,8-octanediol & 18000 & 1.4 & 76 & 67.9 & 125 & 13.06 & 0.453 \\
\hline \multirow{2}{*}{ 1,9-nonanediol } & \multirow{2}{*}{9500} & \multirow{2}{*}{2.5} & \multirow{2}{*}{68} & \multirow{2}{*}{75.6} & \multirow{2}{*}{147} & 14.38 & 0.411 \\
\hline & & & & & & 16.04 & 0.369 \\
\hline \multirow{2}{*}{ 1,10-decanediol } & \multirow{2}{*}{8500} & \multirow{2}{*}{2.5} & \multirow{2}{*}{72} & \multirow{2}{*}{80.4} & \multirow{2}{*}{142} & 14.38 & 0.411 \\
\hline & & & & & & 16.03 & 0.369 \\
\hline 1,12-dodecanediol & 5000 & 2.5 & 84 & 04.1 & 100 & 16.01 & 0.369 \\
\hline
\end{tabular}

In order to expand the polymerization scope, these non-eutectic acid base catalysts were investigated for the homopolymerization of a series of aliphatic diols with various number of methylene units. For this purpose, we investigated the homopolymerization of 8 different diols to synthesize the corresponding polyethers. Thus, 1,4-butanediol, 1,5-pentanediol, 1,7-heptanediol, 1,8-octanediol, 1,9-nonanediol, 1,10-decanediol, 1,11-undecanediol and 1-12-dodecanediol, were polymerized under the optimized conditions previously described for 1-6-hexanediol (Scheme 1).

The polymerizations of diols having methylene units from 6 to 12 were confirmed using ${ }^{1} \mathrm{H}$ NMR and SEC (SI section). The ${ }^{1} \mathrm{H}$ NMR results were similar for all the polyethers, obtaining high conversion in all cases. Regarding SEC results, the polyethers had molecular weights between 5000 to $22000 \mathrm{~g} \mathrm{~mol}^{-1}$ and the dispersity values obtained were between 1.9 and 2.5, values that are slightly high for polycondensation reactions. $\mathrm{x}=4,5,6,7,8,9,10,11,12 \quad 130^{\circ} \mathrm{C}-180^{\circ} \mathrm{C}-200^{\circ} \mathrm{C}$

$\left[\mathrm{O}-\left(-\mathrm{CH}_{2}\right)\right]_{\mathrm{n}} \mathrm{OH}+(\mathrm{n}-1)^{\uparrow} \mathrm{H}_{2} \mathrm{O}$

\footnotetext{
${ }^{a}$ Determined by SEC in $\mathrm{CHCl} 3,{ }^{b}$ Isolated yield,${ }^{c}$ Measured by DSC, ${ }^{d}$ Obtained byWAXS.
} 


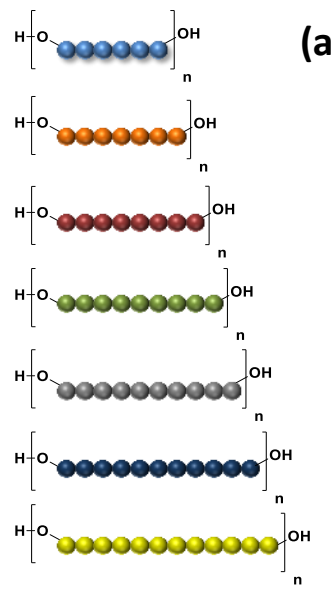

(a)

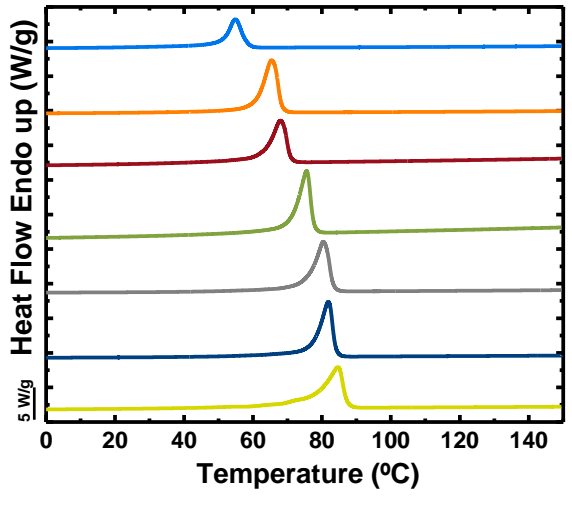

(b)

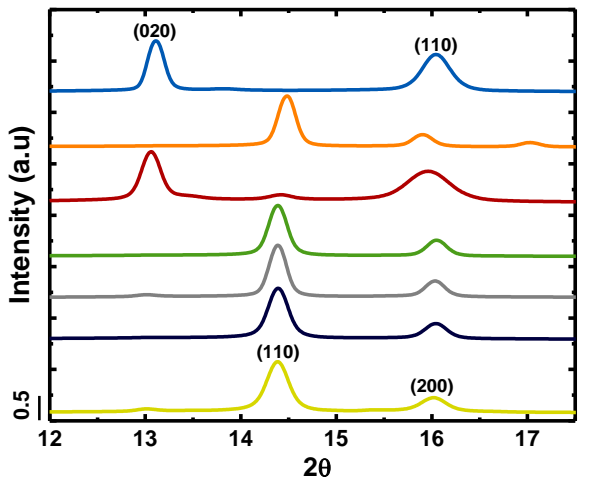

Figure 3 a) DSC heating scans of the linear polyethers and b) WAXS diffraction patterns at $25^{\circ} \mathrm{C}$.

Although all polymerizations were similar, there is a discret trend between the number of the methylene units and the molecular weight. The polymerization was not successful in the cases of 1,4-butanediol and 1,5-pentanediol. The ${ }^{1} \mathrm{H}$ NMR spectra obtained from the self-condensation of 1,5-pentanediol revealed that no signal attributed to the ending group expected at $3.65 \mathrm{ppm}$ was observed, and only the signal of the protons attributed to the signal adjacent to the ether bound was observed (Figure S7). This event suggests that cyclic ethers were obtained instead of linear polymers as it was also reported by Fadret et al. This fact could be associated with the ability of small diols to promote intramolecular etherification giving rise to highly stable 5 and 6 membered cyclic ethers which were removed by the high-vacuum conditions instead of the polyetherification reaction. ${ }^{13} \mathrm{As}$ previously indicated, the molecular weight decreased with the number of methylene units in the repeating unit. We believe that this fact could be attributed to the higher viscosity of the systems as the number of methylene units increases which limited the diffusion of the water and decreases the molecular weight. This has been recently reported in the polycondensation polymerizations of aliphatic polycarbonates. ${ }^{3}$ To exclude the possible cycle formation, MALDITOF analysis were performed and only a telechelic polymer end-capped with alcohol linear species were detected separated by $100 \mathrm{~g} \mathrm{~mol}^{-1}$ (molecular weight of the poly(oxyhexamethylene) repeating unit), attesting the presence of linear species (SI).

The thermal characteristics of the different aliphatic polyethers were measured by DSC (Figure 3a). From DSC curves, the melting $\left(T_{m}\right)$ and crystallization temperatures $\left(T_{c}, \mathrm{SI}\right)$ were determined for all samples. At a first glance, the results revealed that all polyethers were semicrystalline showing $T_{m}$ values between 54 and $85{ }^{\circ} \mathrm{C}$. All thermal transitions are a function of the number of methylenic units along the chain. As the aliphatic chain increases in length (i.e., the number of $\mathrm{CH}_{2}$ units increases in the chains), the first order thermal transitions of crystallization and melting increase in temperature. At the same time, the latent heats of enthalpy and fusion also increase slightly with the number of methylenic units along the repeating unit of the polyether chains. On the other hand, due to the high degree of crystallinity in these aliphatic polyethers $T_{g}$ determinations by DSC were not possible.

The crystal structure of the prepared polyethers was examined by WAXS performed at the synchrotron with a wavelength of 1.0 A. X-ray diffraction measurements showed that polyethers with 6 and 8 methylene units have a different crystal structure in comparison to polyethers with 7,9,10,11 and 12 methylene units (Figure 3b). On the polyether series with 6 and 8 methylene units very strong (020) and strong (110) reflections are observed at $2 \theta$ values of $13.1^{\circ}$ and $16^{\circ}$, respectively, which correspond to monoclinic unit cells, similar to the ones reported for polytetrahydrofurane. ${ }^{12}$ For polyethers with 9 to 12 methylene units, the crystalline structure is completely different and the reflections associated with (110) and (200) planes appeared at $2 \theta$ values of $14.3^{\circ}$ and $16^{\circ}$ respectively which are closely similar to the ones reported for the orthorhombic polyethylene unit cell. In the case of poly(1,7-heptanediol) the crystalline form is not related to either of these previous two structures, however its lateral molecular arrangement is quite similar to orthorhombic polyethylene. Table 2 shows the diffraction spacings $(d)$ calculated according to Bragg's Law. These results are consistent with the crystalline structures reported for polyethers with identical chemical structures but synthesized by different methods. ${ }^{12}$

\section{Synthesis of aliphatic copolyethers and polyether thermo- sets}

In order to broaden the described strategy, copolymerization between different diols was also considered. The synthesis of random copolymers is a good way to modulate the properties and the arquitecture of homopolymers through selecting functional monomers or varying the copolymer composition. ${ }^{21,22}$ 


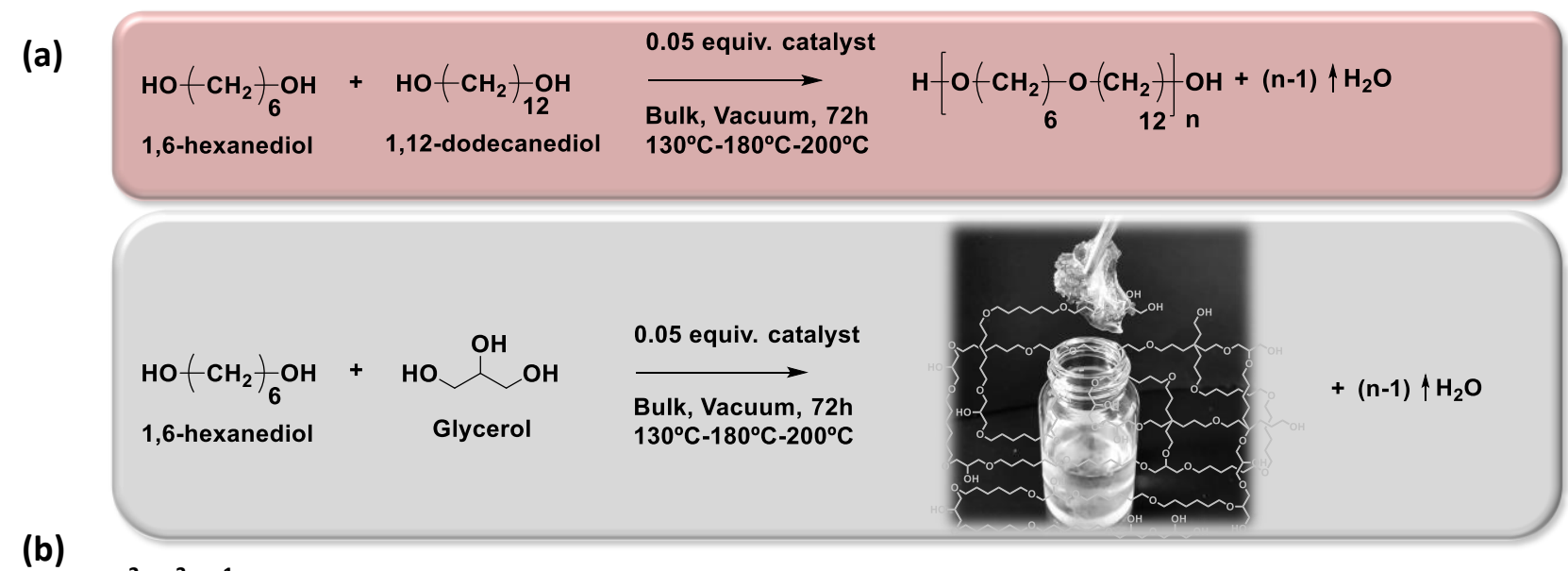

(b)

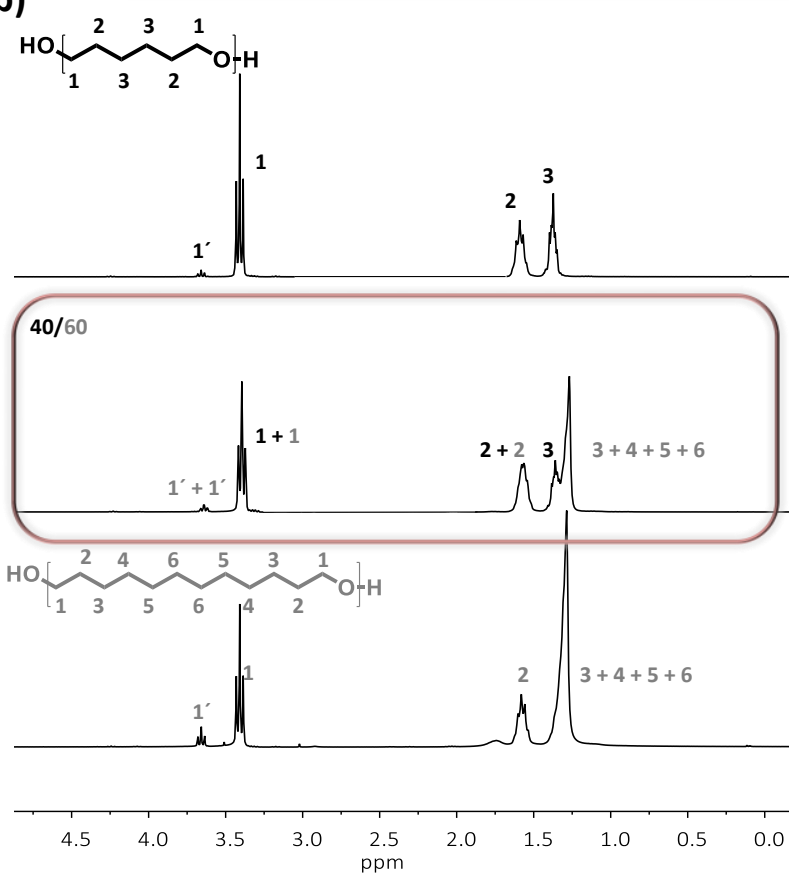

(c)

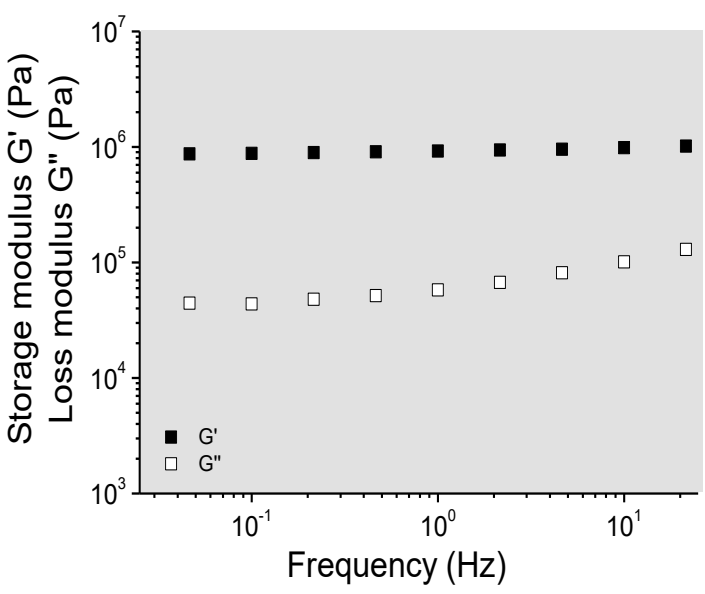

Figure 4. a) Synthesis route of random copolyethers from two different diol monomers, b) ${ }^{1} \mathrm{H}$ NMR spectra of a) poly(oxyhexamethylene), b) copolyether 1,6/1,12 40/60 and c) poly(oxydodecamethylene and c) Dynamic mechanical analysis of the polyetherthermosets.

Thus, diols with different length (1,6-hexanediol and 1,12-dodecanediol) were copolymerized in a 50/50 molar ratio and 1,6 hexanediol was also copolymerized with an alcohol with mutliple functionality such as glycerol.The copolyethers were synthetized under the previously reported conditions. The copolymer $1,6 / 1,12$ was purified and characterized by ${ }^{1} \mathrm{H}$ NMR spectroscopy (Figure 4b). The polyether formation was confirmed following the appearance of the signal 1 at $3.40 \mathrm{ppm}$ attributed to the ether linkage $\left(-\mathrm{CH}_{2}-\mathrm{O}-\mathrm{CH}_{2}-\right)$. The molar composition of the copolyether was determined by ${ }^{1} \mathrm{H}$ NMR according to the equation presented in the SI section. We found that the copolymer composition of 1,6-hexanediol/1,12-dodecanediol resulted in 40/60 mol \%. This composition differed slightly from the initial feed probably due to partial evaporation of 1,6 hexanediol during the copolymerization. The copolymer was also characterized by ${ }^{13} \mathrm{C}$ NMR spectroscopy and the presence of dyads in the copolymer chain was observed (SI). The molecular weight and dispersities of the copolymer determined by SEC analysis was similar to the ones of the homopolymers $9800 \mathrm{~g} \mathrm{~mol}^{-1}$ with a Đ of 2.3.

When 1-6-hexanediol was copolymerized with glycerol a crosslinked unsoluble polymer was obtained. Due to the three hydroxy groups of glycerol and the high \% of glycerol used in feed polyether thermosets were obtained in this case. In order to confirm the polyether formation FT-IR spectra of the resulting unsoluble polymer was acquired (Figure SI). The broad peak around $3000 \mathrm{~cm}^{-1}$, attributed to the $-\mathrm{OH}$ stretching from the hydroxyl group significantly reduced its intensity. Whereas a new band characteristic to the C-O-C stretching vibration at 1050 $\mathrm{cm}^{-1}$ appreared confirming the formation of ether bonds.

The gel formation was confirmed by frequency sweep experiments in compression mode. As observed in Figure 4c, the material shows a predominant elastic behaviour ( $\mathrm{G}^{\prime}>\mathrm{G}^{\prime}$ ') with an elastic modulus value G' 106 Pa. In addition, both $\mathrm{G}^{\prime}$ and $\mathrm{G}$ ', values were independent of frequency, as it corresponds to a 
chemically cross-linked networks. This cross-linked polyether is represented in the image of the Figure $4 \mathrm{a}$.

\section{Conclusion}

Here we report a bulk polycondensation method at relatively high temperature and using simple and recyclable organocatalysts for the production of aliphatic polyethers of medium and large number of methylene units. The molecular structure of the non-eutectic acid base complexes formed between the nonequimolar mixtures of MSA:TBD were characterized using ${ }^{1} \mathrm{H}$ NMR confirming the presence of a unique compound. This fact was confirmed using DFT calculations. Furthermore, under optimized conditions a series of polyethers with different molecular weights from diols with 6 to 12 methylene units were polymerized using the non-eutectic acid base mixture MSA:TBD 3:1. Semicrystalline polyethers with molecular weights between 5000 and $22000 \mathrm{~g} \mathrm{~mol}^{-1}$ were obtained. The polyethers showed melting temperatures between 55 and $85{ }^{\circ} \mathrm{C}$ which increased with the number of methylene units. Two types of crystal structures were found, the monoclinic polytetrahydrofuran type (for 6 and 8 methylene units) and the orthorhombic polyethylene type (for 7,9,10,11 and 12 methylene units). Moreover, we expanded the self-condensation for the preparation of valueadded copolymers from different length chain diols and different functionality, giving rise to different copolymer architectures from linear copolyethers to polyether thermosets. This economic and sustainable synthesis strategy reveals a potential green approach to produce polyethers, copolyethers and thermosets, wich can be applied from low temperature thermosets to new soft segments for polyurethane chemistry.

\section{ASSOCIATED CONTENT}

\section{Supporting Information.}

Experimental section including materials and methods, ${ }^{1} \mathrm{H}$ and ${ }^{13} \mathrm{C}$ NMR spectra, SEC, DSC, WAXS, TGA, MALDI, diffusion coefficients and Density functional theory (DFT) calculation data.

\section{AUTHOR INFORMATION}

\section{Corresponding Author}

Haritz Sardon. E-mail: haritz.sardon@ehu.es

\section{Author Contributions}

The manuscript was written through contributions of all authors. / All authors have given approval to the final version of the manuscript.

\section{Notes}

The authors declare no competing financial interest.

\section{ACKNOWLEDGMENT}

The authors thank the European Commission for its financial support through the projects SUSPOL-EJD 642671. Haritz Sardon and David Mecerreyes gratefully acknowledge financial support from MINECO through project POLYCE. A. J. Müller, O. Coulembier and $\mathrm{H}$. Sardon also acknowledge European funding by the RISE BIODEST project (H2020-MSCA-RISE-2017-778092). The authors also thank the technical and human support provided by Mrs. Sofia Guezala (SGIker) of UPV/EHU for the NMR analysis. H. Sardon, A.J. Müller and I. Flores acknowledge funding and beam time from ALBA Synchrotron facility thorugh the project: 2017092338 (2018). O.C is Research Associate for the F.R.S.FNRS.

\section{REFERENCES}

Klein, R.; Wurm, F. R. Aliphatic Polyethers: Classical Polymers for the 21st Century. Macromol. Rapid Commun. 2015, 36 (12), 1147-1165.

Alkattan, M.; Prunet, J.; Shaver, M. P. Functionalizable Stereocontrolled Cyclopolyethers by Ring-Closing Metathesis as Natural Polymer Mimics. Angew. Chem. Int. Ed. 2018, 57 (39), 12835-12839.

Meabe, L.; Lago, N.; Rubatat, L.; Li, C.; Müller, A. J.; Sardon, H.; Armand, M.; Mecerreyes, D. Polycondensation as a Versatile Synthetic Route to Aliphatic Polycarbonates for Solid Polymer Electrolytes. Electrochimica Acta 2017, 237, 259-266.

Engels, H.-W.; Pirkl, H.-G.; Albers, R.; Albach, R. W.; Krause, J.; Hoffmann, A.; Casselmann, H.; Dormish, J. Polyurethanes: Versatile Materials and Sustainable Problem Solvers for Today's Challenges. Angew. Chem. Int. Ed. 2013, 52 (36), 9422-9441.

Knop, K.; Hoogenboom, R.; Fischer, D.; Schubert, U. S Poly(ethylene Glycol) in Drug Delivery: Pros and Cons as Well as Potential Alternatives. Angew. Chem. Int. Ed. 2010, 49 (36), 6288-6308.

Perry, S.; Hibbert, H. Studies on Reactions Relating to Carbohydrates and Polysaccharides. LXI. The Mechanism of Polymerization of Ethylene Oxide ${ }^{1}$. J. Am. Chem. Soc. 1940, 62 (10), 2599-2604.

Vandenberg, E. J. Organometallic Catalysts for Polymerizing Monosubstituted Epoxides. J. Polym. Sci. 1960, 47 (149), 486-489.

Dreyfuss, M. P.; Dreyfuss, P. A "living" Polymer after Cationic Initiation. Polymer 1965, 6 (2), 93-95.

Uhrich, K. E.; Hawker, C. J.; Frechet, J. M. J.; Turner, S. R. One-Pot Synthesis of Hyperbranched Polyethers. Macromolecules 1992, 25 (18), 4583-4587.

Dannecker, P.-K.; Biermann, U.; von Czapiewski, M.; Metzger, J. O.; Meier, M. A. R. Renewable Polyethers via GaBr3Catalyzed Reduction of Polyesters. Angew. Chem. Int. Ed. . 2018, 57, (28), 8775-8779.

Rhoad, M. J.; Flory, P. J. The Synthesis of Polymeric Ethers. J. Am. Chem. Soc. 1950, 72 (5), 2216-2219.

Kobayashi, S.; Tadokoro, H.; Chatani, Y. Structural Studies on Polyethers, [-(CH2)m-O-]n. VI. The Higher Members with $\mathrm{M}=6-10,12$. Makromol. Chem. 1968, 112 (1), 225-241.

Zhang, S.; Féret, A.; Lefebvre, H.; Tessier, M.; Fradet, A. Poly(oxyalkylene) Synthesis in Brønsted Acid Ionic Liquids. Chem. Commun. 2011, 47 (39), 11092-11094.

Jehanno, C.; Flores, I.; Dove, A. P.; Muller, A.; Ruipérez, F.; Sardon, H. Organocatalysed Depolymerisation of PET in a Fully Sustainable Cycle Using Thermally Stable Protic Ionic Salt. Green Chem. 2018, 20, 1205-1212.

Flores, I.; Demarteau, J.; Müller, A. J.; Etxeberria, A.; Irusta, L.; Bergman, F.; Koning, C.; Sardon, H. Screening of Different Organocatalysts for the Sustainable Synthesis of PET. Eur. Polym. J. 2018, 104, 170-176.

García-Argüelles, S.; García, C.; Serrano, M. C.; Gutiérrez, M. C.; Ferrer, M. L.; Monte, F. del. Near-to-Eutectic Mixtures as Bifunctional Catalysts in the Low-Temperature-RingOpening-Polymerization of $\varepsilon$-Caprolactone. Green Chem. 2015, 17 (6), 3632-3643.

Frisch, M.; Trucks, G.; Schlegel, H.; Scuseria, G.; Robb, M.; Cheeseman, J.; Scalmani, G.; Barone, V.; Mennucci, B.; Petersson, G.; et al. Gaussian 09, Revision B.01; 2009.

Kelly, J. T.; Knorke, H.; Asmis, K. R. Isolating the Isomeric Hydrogen Bonding Signatures of the Cyanide-Water Complex by Cryogenic Ion Trap Vibrational Spectroscopy. $J$. Phys. Chem. Lett. 2017, 8 (21), 5349-5354.

Yang, H.; Zhang, J.; Li, J.; Jiang, S. P.; Forsyth, M.; Zhu, H. Proton Transport in Hierarchical-Structured Nafion Membranes: A NMR Study. J. Phys. Chem. Lett. 2017, 8 (15), 3624-3629.

Sardon, H.; Engler, A. C.; Chan, J. M. W.; García, J. M.; Coady, D. J.; Pascual, A.; Mecerreyes, D.; Jones, G. O.; Rice, J. 
E.; Horn, H. W.; Hedrick, J. L. Organic Acid-Catalyzed Polyurethane Formation via a Dual-Activated Mechanism: Unexpected Preference of N-Activation over O-Activation of Isocyanates. J. Am. Chem. Soc. 2013, 135 (43), 16235-16241.

(21) Zhang, H.; Grinstaff, M. W. Recent Advances in Glycerol Polymers: Chemistry and Biomedical Applications. Macromol. Rapid Commun. 2014, 35 (22), 1906-1924.

(22) He, J.; Burt, S. P.; Ball, M.; Zhao, D.; Hermans, I.; Dumesic, J. A.; Huber, G. W. Synthesis of 1,6-Hexanediol from Cellulose Derived Tetrahydrofuran-Dimethanol with PtWOx/TiO2 Catalysts. ACS Catal. 2018, 8 (2), 1427-1439. 


\section{For Table of Contents Use Only}

We disclose a bulk polycondensation method at high temperature and using a simple non-eutectic acid base organocatalysts for the production of aliphatic polyethers.

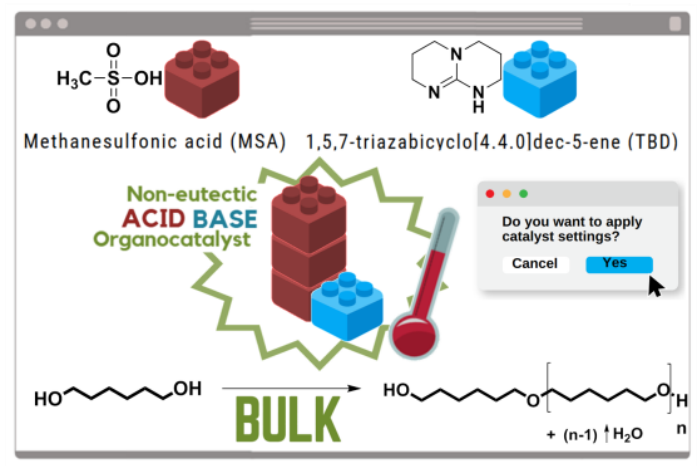

